\title{
A NEW AUTOMATIC ON-BOARD MULTISPECTRAL IMAGE COMPRESSION SYSTEM FOR LEO EARTH OBSERVATION SATELLITES
}

\author{
Guoxia Yu, Tanya Vladimirova and Martin Sweeting \\ Surrey Space Centre, School of Electronics and Physical Sciences \\ University of Surrey, GU2 7XH, UK \\ \{g.yu, t.vladimirova, m.sweeting\}@ surrey.ac.uk
}

\begin{abstract}
This paper has proposed an automatic onboard multispectral image compression system, for LEO Earth Observation satellites. This system has combined tiling, sub-pixel band registration, radiometric calibration, and multispectral image compression, to achieve automatic on-board image processing requirement. Two radiometric calibration methods are compared, and Brightness Difference Compensation (BDC) is preferred. A new robust band registration technique, which is gradient image based phase correlation (GradPC), is proposed. Experimental work based on natural satellite test images is given and results validate the system design.
\end{abstract}

Index Terms - Multispectral image compression, automatic, on-board, band registration, radiometric calibration

\section{INTRODUCTION}

Earth Observation Satellites require transmission to ground of an extensive amount of imaging data. The data transmission capacity of onboard equipment these days is reaching several times of the down link circuit capacity provided by present satellites. Data compression is believed to be a solution to the "Bandwidth Versus Data Volume" dilemma of modern spacecraft.

Surrey Satellite Technology Ltd. (SSTL), with a majority share owned by University of Surrey, is the global leader in small satellite design and manufacturing. The Disaster Monitoring Constellation (DMC), developed by SSTL, consist five low-cost small satellites, which belong to participating countries: United Kingdom, Nigeria, Algeria, Turkey and China. From LEO, each satellite provides 32 metre, multispectral imaging, over a $600 \mathrm{~km}$ swath width. The DMC program offers the possibility for daily revisiting of any point on the globe [1].

Regarding band registration, phase correlation method, based on the Fourier Shift Theorem, shows strong robustness against noises and non-uniform disturbances. The first band registration system was proposed in [4]. The gradient representation of image is threshold to produce a binary boarder map, and then a registration method, similar with phase correlation is applied on the edge map. An edgebased correlation image registration algorithm called EdgeReg was presented in [5], which includes an initial edge-filtering stage before phase correlation. Images are gradient filtered on a band-by-band basis using a simple Sobel-like edge detection method. However, these edgebased techniques are very sensitive to the threshold used, which will lead to erratic registration results. A new technique based on gradient image for robust band registration is proposed.

For current onboard compression systems [2], multispectral images are mostly processed band-by-band, which is called intra-band compression. In this case, spectral redundancy still exists in compressed file. Spectral redundancy, which lies specifically in multispectral images, can be removed via spectral decorrelation. Although spectral decorrelation has been actively investigated in the literature has not yet been applied to on-board multispectral image compression. This paper effectively puts on-board multispectral image compression into practice, based on the survey, development trends, and the proposed system presented in [2].

In the following section, the proposed on-board image compression system is introduced. Section 3 will present practical results, using DMC multispectral test images. And conclusions are given in Section 4.

\section{A NEW PROPOSED ON-BOARD IMAGE COMPRESSION SYSTEM}

The architecture of the proposed on-board image compression system is shown in Figure 1. The image data, after coming out of the cameras, is processed serially, tileby-tile, first undergoing some pre-processing tasks, which improves compression performance or enables the systems to make intelligent decisions about the compression process.

\subsection{Tiling}


The primary reason to do tiling is that the dimension of satellite image is generally awfully large. It is too complicated to handle with the whole image. On the other hand, tiling scheme constrains error propagation in one tile, a small area. However the size of one tile can't be very small, otherwise compression performance will be degraded, due to less decorrelation ability and more subsidiary data. The required buffer memory to store image data temporarily is proportional to tile size, so it can't be very large either.

\subsection{Radiometric calibration}

Radiometric calibration is defined as the process of converting Digital Number (DN) of raw image data to radiance quality that went into sensor. A simple radiometric calibration, abbreviated to SRC, consists of gain correction and offset removing, as shown Equation 1. One can see vertical strips are removed by radiometric calibration in Figure 2.

$$
P V=\text { gain } \times D N-\text { bias }
$$

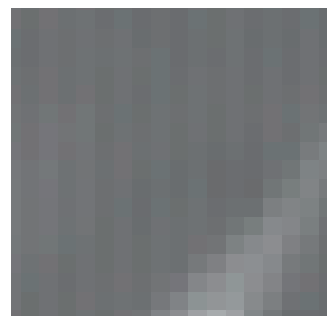

(a) Pan

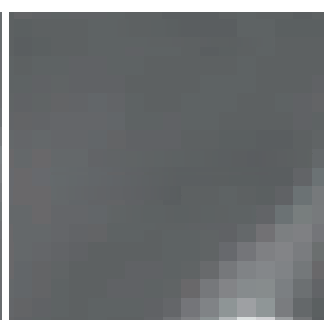

(b) Pan-R
Figure 2 Part of one Beijing-1 panchromatic test image with (a) PAN - before radiometric calibration; (b) PAN-R - after radiometric calibration

An alternative method, Brightness Difference Compensation (BDC) is considered here, which was proposed and originally called Integer Gamma Difference (IDG) compensation in [3]. By the compensation of brightness difference, image is expected to have vertical strips removed. This process is exactly lossless. While SRC with floating point computation isn't.

\subsection{Band registration}

A new robust band registration technique is proposed here. Inspired by [6], derived gradient image is fed to phase correlation, instead of original image or its edge map. But only the magnitude information is used here, and the definition of gradient is modified and shown in Equation 2. The gradient value is the sum of absolute value of difference in vertical and horizontal direction. But the interval is two pixels. This proposed gradient image based phase correlation, is abbreviated to GradPC.

$$
\operatorname{Grad}=\left|F_{i+1, j}-F_{i-1, j}\right|+\left|F_{i, j+1}-F_{i, j-1}\right|
$$

Extension to sub-pixel registration has been achieved simply by the calculation of two-dimensional centre of mass, around a small area of the peak found in previous stage.

\subsection{Spectral decorrelation}

Karhunen-Loeve Transform (KLT), which is considered as the optimum method to spectrally decorrelate MS data [7], is an active research topic recently. In [8], a fixed data rate compressor for multispectral image is utilizing KLT to decorrelate image data. By KLT, each band is transformed into Eigen plane. Then each Eigen plane is mapped into Eigen image, by linear shrink if its dynamic range is greater than source image's.

\subsection{Image compression}

For two-dimensional image compression part, Vladimirova [3] reached such suitable choices as, JPEG-LS for lossless compression, JPEG2000 for lossy compression. Consultative Committee for Space Data Systems (CCSDS) produced an image data compression (IDC) recommendation suitable for space applications in November 2005. Its architecture is similar to that of JPEG2000. According to the blue book and other literature sources [9] CCSDS-IDC could achieve performance similar to JPEG2000, while having less complexity.

\subsection{Selective image compression}

Selective image compression is one of important development trends. It normally requires change detection or classification. First if no change detected at all, this image doesn't need to be saved or downloaded. If any changes detected, then only the change part needs to be processed. On the other hand, after classification is finished, regions classified into certain classes are labelled as region of interest, while regions with others classes are labeled as region of non-interest. Compression algorithms will treat these regions differently. Works on Change detection and classification are detailed in [10-11].

\subsection{Image content encryption}

Several researchers have recently successfully downloaded unauthorized satellite data directly from passing-by satellites, which has illustrated the need for on-board encryption in imaging payloads. Encryption has been adopted on SPOT-5 and GEZGIN-2. Therefore here a data encryption module [12] is added to assure image data confidential. 


\section{EXPERIMENTAL RESULTS}

We choose DMC multispectral imager as the study case, which have $32 \mathrm{~m}$ GSD and 3 bands in the standard LANDSAT bands 2, 3 and 4 (Green, Red, and Near Infrared). Test images are composed from multispectral images captured by UK-DMC satellite.

Here 512 by 512 has been decided for one tile. The required buffer memory is around 30 Mbytes, which is acceptable, while each tile is big enough to get competitive compression performance.

Comparison of no radiometric calibration, SRC, and $\mathrm{BDC}$, on lossless compression is made, with results shown in Table 1. With radiometric calibrations, compression ratio is improved by around $5.5 \%$. BDC, which gives best compression performance, hence is preferred.

Table 1 Compression results of JPEG-LS with no radiometric calibration, SRC, and BDC, on Beijing-1 panchromatic test images

\begin{tabular}{|l|l|l|l|}
\hline CR & JPEG-LS & SRC+JPEG-LS & BDC+JPEG-LS \\
\hline DC000055hp & 4.09 & 4.18 & 4.23 \\
\hline DC000138hp & 5.57 & 5.7 & 6.24 \\
\hline DC000189hp & 3.52 & 3.56 & 3.64 \\
\hline DC000219hp & 4.3 & 4.51 & 4.82 \\
\hline DC000231hp & 3.01 & 3.03 & 3.08 \\
\hline DC000285hp & 3.59 & 3.69 & 3.69 \\
\hline DC000286hp & 3.19 & 3.24 & 3.29 \\
\hline DC000288hp & 4.04 & 4.13 & 4.21 \\
\hline DC000305hp & 3.05 & 3.05 & 3.10 \\
\hline AVE & 3.82 & 3.9 & 4.03 \\
\hline
\end{tabular}

The proposed GradPC is much robust, as the failure rate dropped to $9.2 \%$ from $48.5 \%$ of pure phase correlation for one UK-DMC multispectral image, which is shown in Figure 3 . The $9.2 \%$ failure cases happen on the tiles which are homogeneous regions (sea water here).

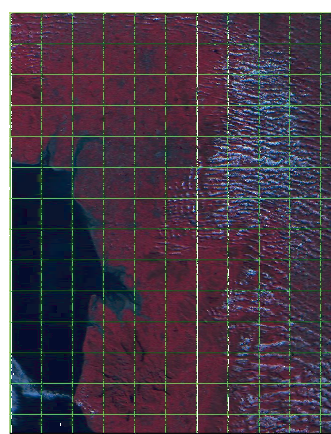

Figure 3 One UK-DMC Multispectral Test Image for Robust Band Registration

Regarding compression performance, multispectral images is processed through tiling, GradPC band registration, BDC radiometric calibration, sub-pixel extension registration, KLT decorrelation, and JPEG2000. Here JPEG2000 is taken, as CCSDS-IDC software hasn't been well developed at the moment. The JPEG2000 software used is Kakadu V5.1.

Test images with different features, such as urban, mountain and agriculture, have been applied to this system. Results on these images yield the same conclusions, so only results of image around urban area are given in this section. Part of this image, in four different cases is shown in Figure 4. One could see each additional step would make the pseudo multispectral image smoother and visually clearer. Using KLT+JPEG2000 as image compression technique, comparison compression results to these four different cases, in terms of PSNR, MAD and PMAD, are shown in Table 2. One can see each step could gain obvious improvement.

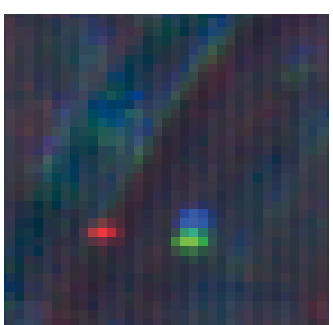

(a)

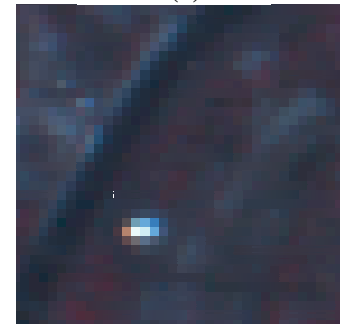

(c)

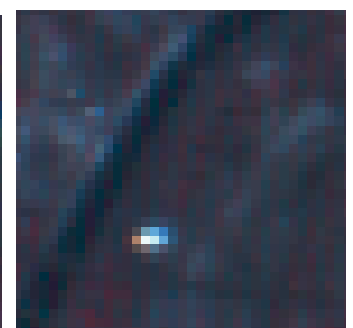

(b)

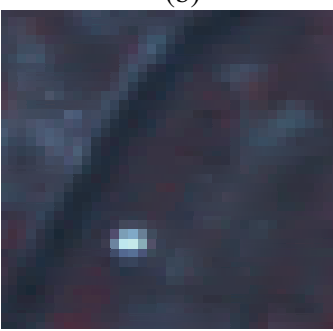

(d)
Figure 4 Part of the urban area test image with (a) raw image; (b) after band registration; (c) after band registration and BDC; (d) after band registration, BDC and sub-pixel registration

Comparison results of intra-band compression (JPEG2000) and inter-band compression (KLT+JPEG2000) on pre-processed image are given in Table 3. KLT spectral decorrelation could achieve number 2 or 3 reduction of MAD.

\section{CONCLUSION}

Proposed GradPC band registration technique is robust. Each pre-processing task is positive, in terms of final compression performance. The proposed system is on its way to be implemented in hardware for real-time on-board application.

\section{REFERENCES}


[1] Surrey Missions: Disaster Monitoring Constellation, Datasheet, 2002, SSTL

[2] G.Yu, T. Vladimirova, M. N. Sweeting. "Image Compression Systems on Board Satellites", Submitted to ACTA ASTRONAUTICA, Journal of the International Academy of Astronautics

[3] T.Vladimirova, A.Steffens. "Compression of Multispectral Images On-Board Observation Satellites" Proceedings of the International Conference "Space, Ecology, Safety" (SES'05), vol.1, pp. 105-110, Varna, Bulgaria, 10-13 June 2005, TU Sofia.

[4] Anuta, P.E., Spatial registration of multispectral and multitemporal digital imagery using fast fourier transform. IEEE transactions on Geoscience Electronics, 1970. 8: p. 353-368

[5] Nandy, P., et al. Edge-based correlation image registration algorithm for the Multispectral Thermal Imager (MTI). in Algorithms and Technologies for Multispectral, Hyperspectral, and Ultraspectral Imagery X. 2004. Orlando, FL, USA: SPIE.

[6] Argyriou, V. and T. Vlachos. Using gradient correlation for sub-pixel motion estimation of video sequences. in Acoustics, Speech, and Signal Processing, 2004.Proceedings. (ICASSP '04). IEEE International Conference on. 2004.
[7] Saghri, J.A.; Tescher, A.G.; Reagan, J.T., "Practical transform coding of multispectral imagery," Signal Processing Magazine, IEEE , vol.12, no.1pp.32-43, Jan 1995

[8] Lambert-Nebout, C., et al. Fixed Data Rate Wavelet Compressor For Multispectral Satellite Systems. in Applications of Digital Image Processing XXV. 2002. Seattle, WA, USA: SPIE.

[9] Pen-Shu Yeh; Armbruster, P.; Kiely, A.; Masschelein, B.; Moury, G.; Schaefer, C.; Thiebaut, C., "The New CCSDS Image Compression Recommendation", 2005 IEEE Conference on Aerospace, pp. 1- 8, 05-12 March 2005

[10] Vladimirova, T., Yuhaniz, S., Meerman, M.J., Stephens, P., Hodgson, D. Intelligent Imaging on Board Small Observation Satellites. Presented at 2006 IEEE International Geoscience and Remote Sensing Symposium. Denver, Colorado. July 31-August 04, 2006.

[11] Hou, P.; Petrou, M.; Underwood, C.I.; Hojjatoleslami, A., "Improving JPEG performance in conjunction with cloud editing for remote sensing applications," Geoscience and Remote Sensing, IEEE Transactions on , vol.38, no.1pp.515-524, Jan 2000

[12] T. Vladimirova, R. Banu, and M. Sweeting, "On-Board Encryption in Satellites", MAPLD 2005, Washington, D.C. Sept. 2005

Table 2 Comparison of compression results at different stages

\begin{tabular}{|l|c|c|c|c|c|c|c|c|c|}
\hline \multirow{2}{*}{ KLT+JPEG2000 } & \multicolumn{3}{|c|}{ PSNR } & \multicolumn{3}{c|}{ MAD } & \multicolumn{3}{c|}{ PMAD } \\
\cline { 2 - 11 } & B1 & B2 & B3 & B1 & B2 & B3 & B1 & B2 & B3 \\
\hline RAW & 40.58 & 37.92 & 38.12 & 13 & 24 & 28 & 0.28 & 0.61 & 0.27 \\
\hline BR & 40.91 & 40.9 & 40.35 & 12 & 14 & 21 & 0.26 & 0.35 & 0.25 \\
\hline BR+BDC & 41.62 & 41.79 & 40.72 & 11 & 14 & 17 & 0.25 & 0.26 & 0.22 \\
\hline BR+BDC+Subpixel & 45.09 & 42.22 & 43.9 & 8 & 12 & 10 & 0.14 & 0.24 & 0.12 \\
\hline
\end{tabular}

Table 3 Comparison of compression results between with and without KLT spectral decorrelation

\begin{tabular}{|l|c|c|c|c|c|c|c|c|c|}
\hline \multirow{2}{*}{ BR+BDC+Subpixel } & \multicolumn{3}{|c|}{ PSNR } & \multicolumn{3}{c|}{ MAD } & \multicolumn{3}{c|}{ PMAD } \\
\cline { 2 - 11 } & B1 & B2 & B3 & B1 & B2 & B3 & B1 & B2 & B3 \\
\hline intra JPEG2000 & 43.93 & 41.22 & 43.11 & 11 & 15 & 12 & 0.17 & 0.31 & 0.16 \\
\hline KLT+JPEG2000 & 45.09 & 42.22 & 43.9 & 8 & 12 & 10 & 0.14 & 0.24 & 0.12 \\
\hline
\end{tabular}

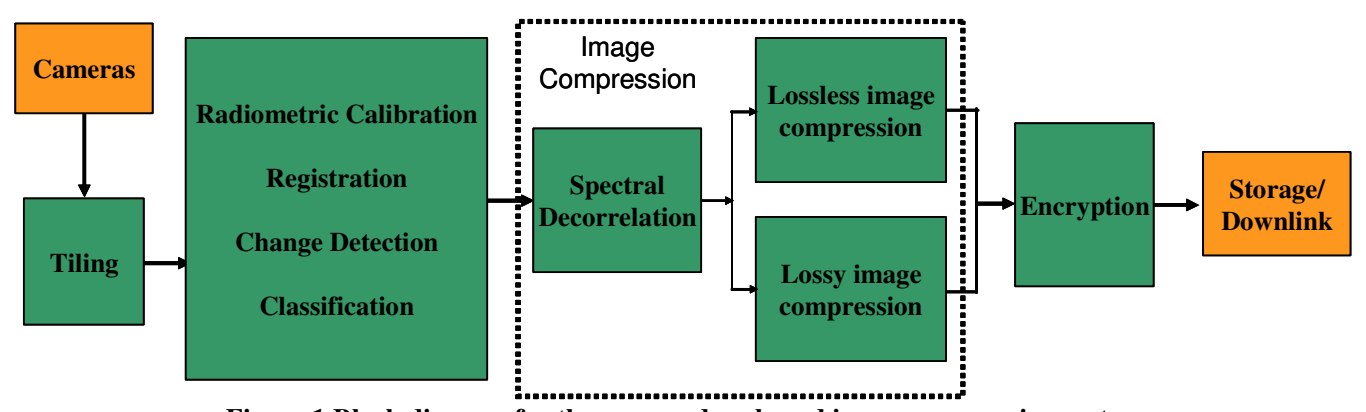

Figure 1 Block diagram for the proposed on-board image compression system 